\title{
Bilateral SUNCT-like headache in a patient with prolactinoma responsive to lamotrigine
}

\author{
Jasna Zidverc-Trajkovic · Svetlana Vujovic • \\ Ana Sundic · Aleksandra Radojicic · \\ Nadezda Sternic
}

Received: 7 May 2009/Accepted: 29 July 2009/Published online: 11 September 2009

(C) Springer-Verlag 2009

\begin{abstract}
Short-lasting unilateral neuralgiform headache with conjunctival injection and tearing (SUNCT) syndrome is a rare trigeminal autonomic cephalalgia. The cases of SUNCT with attacks that affected both sides simultaneously have only rarely been reported and some of them had underlying pathology. We have reported a case of bilateral SUNCT-like headache secondary to a prolactinoma and responsive to lamotrigine treatment.
\end{abstract}

Keywords SUNCT syndrome · Prolactinoma · Lamotrigine

\section{Introduction}

Short-lasting unilateral neuralgiform headache with conjunctival injection and tearing (SUNCT) syndrome is an uncommon form of trigeminal autonomic cephalalgia (TAC). Diagnostic criteria for SUNCT syndrome require at least 20 attacks of unilateral orbital, supraorbital or temporal stabbing or pulsating pain lasting 5-240 s and occurring with a frequency from 3 to 200 per day. Probable SUNCT is the syndrome with attacks fulfilling all but one of the criteria for SUNCT [1]. Several cases of secondary SUNCT syndrome, especially in patients with prolactinomas and growth-hormone secreting tumors, have been

J. Zidverc-Trajkovic ( $₫) \cdot$ A. Sundic · A. Radojicic .

N. Sternic

Institute of Neurology, Clinical Centre of Serbia,

Belgrade, Serbia

e-mail: jzidverc@gmail.com

S. Vujovic

Institute of Endocrinology, Clinical Centre of Serbia,

Belgrade, Serbia reported [2]. Although SUNCT syndrome is uncommon and its true frequency is unclear, its presence in nearly $5 \%$ of patients in the cohort with headache and pituitary tumors suggests that this syndrome may be over-represented in pituitary disease [2].

According to the existing results, lamotrigine is the treatment of choice in SUNCT, followed by topiramate and gabapentin as proposed by EFNS Task Force [3].

We have reported the case of headache fulfilling all diagnostic criteria for SUNCT syndrome except the criterion of unilateral presentation, associated with microprolactinoma and highly responsive to lamotrigine.

\section{Case report}

We report a case of a 24-year-old right-handed female presented with a 6-year history of headaches in March 2006. A typical attack was bilateral with pain affecting both sides simultaneously. The pain with an electric shock-like quality was centered on the retro-orbital region and temple with radiation to the occipital regions. The attacks had high pain intensity, estimated by the patient as $9-10$ on a verbal analogue scale (0-10), lasting $30 \mathrm{~s}$ and was associated with prominent conjunctival injection, lacrimation and periorbital face flushing that occurred five to ten times a day, and attacks of moderate pain, estimated as 6 on the same scale that lasted 5-6 s and occurred every 15-20 min. She had attacks only when awake, with the first starting an hour after awaking, and was completely pain free during sleep. Head movements, talking, eating, and blowing nose or other manipulations could not trigger the attacks.

The patient had previously tried aspirin, diclofenac, paracetamol/caffeine combinations, amitriptyline $75 \mathrm{mg}$ daily, sodium valproate $1,000 \mathrm{mg}$ daily, all of which were 
ineffective. Carbamazepine $800 \mathrm{mg}$ daily reduced the frequency of milder attacks for about $30 \%$ and had no effect on attacks of higher intensity.

Previous medical history was unremarkable. There was no history of headaches in her family. She had regular menstrual cycles, denied galactorrhea or problems during sexual intercourse.

A detailed general and neurological examination was normal. The observations revealed that the conjunctival injection, lacrimation and face flushing were more prominent during more severe attacks. In spite of the patient's statement that pain is simultaneous and had equal intensity on both sides, we have observed that the autonomic features were more prominent on the right side. Routine hematological and biochemical screening were normal.

A trial of high-dose oxygen inhalation at flow rate $15 \mathrm{l} /$ min and subcutaneous sumatriptan $6 \mathrm{mg}$ had no effect. Indomethacin administered in three doses of $25 \mathrm{mg}$ per day was ineffective, as well as further dose increment that stopped at daily dose of $125 \mathrm{mg}$ because of constant dull headache.

According to IHCD-II criteria [1], a diagnosis of probable SUNCT syndrome was made. Gabapentin at daily dose of 1,200 mg was ineffective. Lamotrigine was started at a dose of $25 \mathrm{mg}$ once daily and the dose was increased to $100 \mathrm{mg}$ twice daily over 6 weeks. During the first month on lamotrigine $200 \mathrm{mg}$ daily the SUNCT attacks were completely suppressed during 1-year follow-up. Trials of dose reducing led to attacks' recurrence.

In spite of the fact that neurological examination was normal and that the headaches resolved completely with lamotrigine therapy, the bilaterality of patient's headache instantly raised the concern about possible symptomatic origin. According to the literature data about frequent association of SUNCT-like headache and pituitary tumors $[2,5-8]$ and recommendations of the Serbian Headache Guideline [4] that "MRI examination should be performed on all patients with TACs because of relative rareness of the disorders with high frequency of secondary cases", MRI scan of pituitary-hypothalamic region was performed.

Magnetic resonance imaging revealed the pituitary microadenoma at the right side of the gland without extending towards the surrounding structures (Fig. 1). The examination of pituitary hormones showed that serum prolactin levels were 2,125 mlU/l (normal range 130700), while the levels of follicle-stimulating hormone, luteinizing hormone, oestradiol, adrenocorticotropin hormone, basal cortisol, thyroid-stimulating hormone, free thyroxin, insulin, and growth factor were within normal range, so multiple endocrine neoplasm was ruled out. The treatment with bromocriptine was started and lamotrigine was suspended. After 2 months of bromocriptine therapy serum prolactin levels were $574 \mathrm{mlU} / \mathrm{l}$ and cycle was ovulatory (progesterone level was $27 \mathrm{nmol} / 1$ on the $21 \mathrm{st}$ day of cycle). However, on bromocriptine therapy and without lamotrigine, she had frequent SUNCT-like attacks of high intensity especially from the 6th to the 13th day of menstrual cycle. The lamotrigine therapy was initiated again. Trials of drug reduction, performed every second month succeed after 6 months with complete headache resolution.
Fig. 1 Magnetic resonance imaging (MRI) scan of the brain revealed a pituitary adenoma on the right side of the gland without extending towards the cavernous sinus

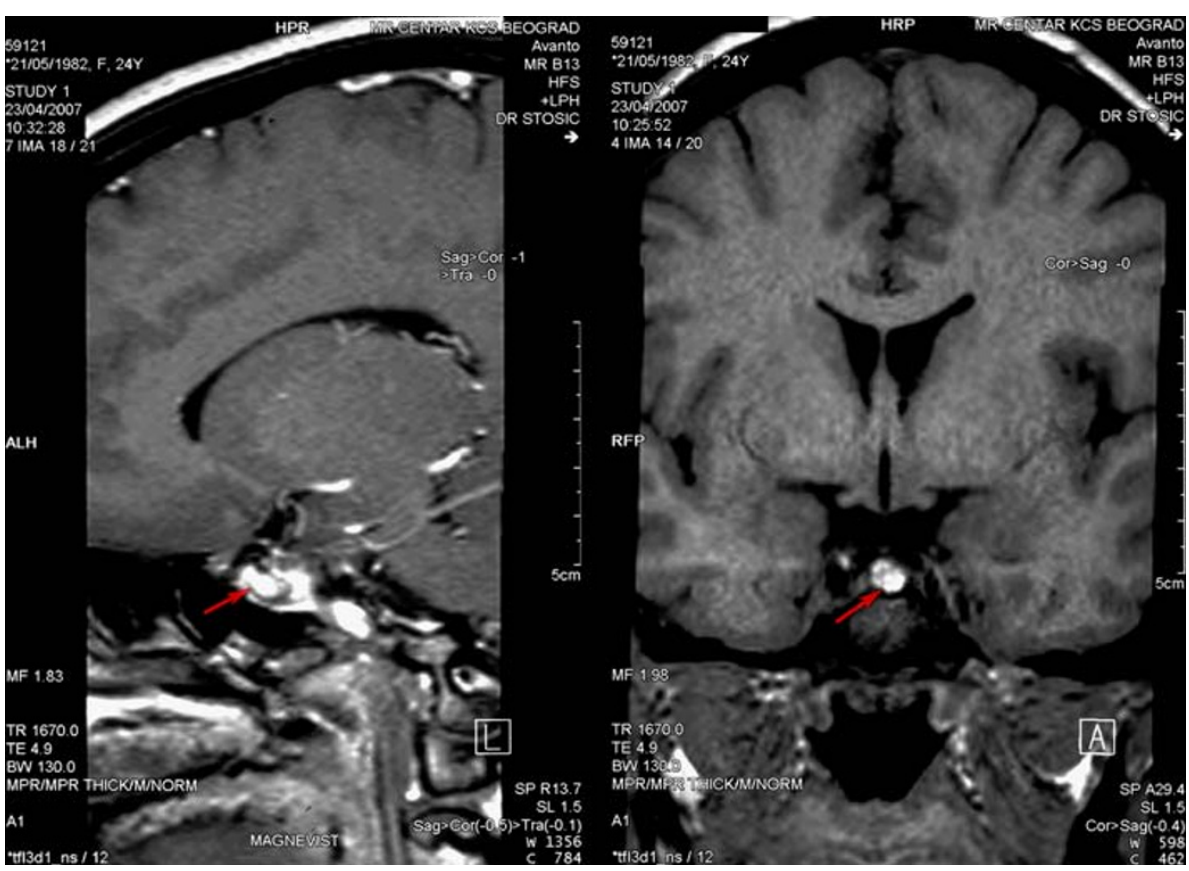




\section{Discussion}

The presented patient with frequent short-lasting attacks of pain associated with conjunctival injection, lacrimation and facial flushing fulfills the criteria for SUNCT syndrome. The attacks of the presented patient were bilateral. As for other TACs, strictly unilateral pain localization in SUNCT syndrome is an obligatory diagnostic criterion [1]. The exceptions from this rule with bilateral pain presentation were described for primary SUNCT [9, 10]. However, bilateral presentation of pain was more frequently observed in symptomatic cases. In large group of patients with pituitary tumors-associated headache, $8 \%$ of them had bilateral pain [2]. Moreover, diagnostic criteria for headaches attributed to hypothalamic or pituitary hyper- or hypo-secretion, coded 7.4.4 by the current classification [1], included bilateral pain presentation.

Incomplete response to carbamazepine, observed in our patient, has been described by other authors, as well [11, 12]. Lamotrigine was very efficacious in the treatment of our patient. The existing evidence favors lamotrigine and, according to a recent drug trial, topiramate [13] as the most effective therapies for SUNCT syndrome [3, 11]. The effectiveness of lamotrigine in our patient with symptomatic headache underlines that good response to therapy does not exclude the need for extensive diagnostic work-up in order to exclude the underlying pathology. In other words, good response to lamotrigine could not be considered as diagnostic criteria for SUNCT, like indomethacin for paroxysmal hemicrania and hemicrania continua.

There was no evidence of tumor extension to the cavernous sinus or surrounding brain structures in the presented patient. The pain had equal intensity, duration, frequency and quality on both sides as reported by the patient. These features support the current opinion that the pituitary-associated headache is predominantly neurohumorally mediated by disruption of the dopamine-prolactin axis in the presence of tumor [14]. After 2 months of bromocriptine therapy, when the prolactin serum levels were within physiological range and cycle became ovulatory, our patient experienced partial improvement. In that time, SUNCT-like headaches were occurring only between 6th and 13th day of menstrual cycle, in follicular phase when the estrogen levels were increased.

It is demonstrated, on animal model, that high levels of estrogen influence gene expression and intracellular signaling regulated by kinase, following the up-regulation of cell receptors on pathways for inflammatory and neuropathic pain [15]. Recent neuropharmacological evidence from a primate model of surgical menopause examining effects in the dorsal raphe of macaques suggests that serotonin synthesis and degradation, as well as the neuronal firing are influenced by estrogen receptor-mediated mechanisms [16]. However, it is worth noting that the autonomic features were more prominent ipsilateral to the side of the tumor. The obvious tumor compression of surrounding tissue was not detected, but the possible local impact on autonomic pathways could not be excluded.

Bromocriptine therapy decreased serum prolactin levels to normal values only after 2 months, while the headaches resolved after 6 months of therapy, requiring simultaneous treatment with lamotrigine. The plausible explanation for the observed time lag and partial efficacy of bromocriptine therapy could be different mechanisms of drug action on dopamine-prolactin axis and trigeminovascular system.

\section{Conflict of interest None.}

\section{References}

1. Headache Classification Subcommittee of the International Headache Society (2004) The International Classification of Headache Disorders. 2nd edn. Cephalalgia 24(Suppl 1):9-160

2. Levy MJ, Matharu MS, Meeran K, Powell M, Goadsby PJ (2005) The clinical characteristics of headache in patients with pituitary tumors. Brain 128:1921-1930

3. May A, Leone M, Afra J, Linde M, Sandor PS, Evers S, Goadsby PJ (2006) EFNS guidelines on the treatment of cluster headache and other trigeminal-autonomic cephalalgias. Eur J Neurol 13:1066-1077

4. Serbian Physicians' Society (2005) Serbian Headache Guideline-National Guidelines for primary care physicians. ISBN 8685313-31-7, pp 22-26

5. Ferrari MD, Haan J, van Seters AP (1988) Bromocriptineinduced trigeminal neuralgia attacks in a patient with a pituitary tumor. Neurology 38:1482-1484

6. Massiou H, Launay JM, Levy C, El Amrani M, Emperauger B, Bousser MG (2002) SUNCT syndrome in two patients with prolactinomas and bromocriptine-induced attacks. Neurology 58:1698-1699

7. Levy MJ, Matharu MS, Goadsby PJ (2003) Prolactinomas, dopamine agonists and headache. Eur J Neurol 10:169-173

8. Matharu MS, Levy MJ, Merry RT, Goadsby PJ (2003) SUNCT syndrome secondary to prolactinoma. J Neurol Neurosurg Psychiatry $74: 1590-1592$

9. Sabatowski R, Huber M, Meuser T, Radbruch L (2001) SUNCT syndrome: a treatment option with local opioid blockade of the superior cervical ganglion? A case report. Cephalalgia 21:154156

10. Kuhn J, Vosskaemper M, Bewermeyer H (2005) SUNCT syndrome: a possible bilateral case responding to topiramate. Neurology 64:2159

11. Cohen A (2007) Short-lasting unilateral neuralgiform headache attacks with conjuctival injection and tearing. Cephalalagia 27:824-832

12. Matharu MS, Cohen AS, Goadsby PJ (2004) SUNCT syndrome responsive to intravenous lidocaine. Cephalalgia 24:985-992

13. Cohen A, Matharu M, Goadsby P (2007) Double-blind placebocontrolled trial of topiramate in SUNCT. Cephalalgia 27:758 
14. Levy MJ, Jager HR, Powell M, Matharu MS, Meeran K, Goadsby PJ (2004) Pituitary volume and headache: size is not everything. Arch Neurol 61:721-725

15. Puri V, Puri S, Svojanovsky S et al (2006) Effects of oestrogen on trigeminal ganglia in culture: implications for hormonal effects on migraine. Cephalalgia 26:33-42
16. Nappi RE, Sances G, Brundu B et al (2005) Estradiol supplementation modulates neuroendocrine response to M-chlorophenylpiperazine in menstrual status migrainous triggered by oral contraception-free interval. Hum Reprod 20:3423-3428 